\title{
Comparison of dynamical degrees for semi-conjugate meromorphic maps
}

Tien-Cuong Dinh and Viêt-Anh Nguyên

\begin{abstract}
Let $f: X \rightarrow X$ be a dominant meromorphic map on a projective manifold $X$ which preserves a meromorphic fibration $\pi: X \rightarrow Y$ of $X$ over a projective manifold $Y$. We establish formulas relating the dynamical degrees of $f$, the dynamical degrees of $f$ relative to the fibration and the dynamical degrees of the map $g: Y \rightarrow Y$ induced by $f$. Applications are given.
\end{abstract}

Mathematics Subject Classification (2010). Primary 37F; Secondary 32U40, 32H50.

Keywords. Semi-conjugate maps, dynamical degree, relative dynamical degree.

\section{Introduction}

Let $\left(X, \omega_{X}\right)$ be a compact Kähler manifold of dimension $k$ and let $f: X \rightarrow X$ be a meromorphic map. We assume that $f$ is dominant, i.e. the image of $f$ contains an open subset of $X$. Let $\pi: X \rightarrow Y$ be a dominant meromorphic map from $X$ onto a compact Kähler manifold $\left(Y, \omega_{Y}\right)$ of dimension $l \leq k$. The fibers of $\pi$ define a fibration on $X$ which might be singular. If $f$ preserves this fibration, i.e. $f$ sends generic fibers of $\pi$ to fibers of $\pi$, it induces a dominant meromorphic map $g: Y \rightarrow Y$ such that $\pi \circ f=g \circ \pi$. In that case, we say that $f$ is semi-conjugate to $g$. For simplicity, we assume that $\omega_{Y}$ is normalized so that $\omega_{Y}^{l}$ is a probability measure.

A natural question is how the dynamical system defined by $f$ is similar to the one defined by $g$ when $f$ is semi-conjugate to $g$ as above. One of the first steps towards understanding this question should be to find out the relations between some invariants associated to $f$ and $g$. In this paper, we will compare their dynamical degrees.

Let $f^{n}:=f \circ \cdots \circ f, n$ times, denote the iterate of order $n$ of $f$. The dynamical degree $d_{p}(f)$ of order $p$ is the quantity which measures the growth of the norms of $\left(f^{n}\right)^{*}$ acting on the Hodge cohomology group $H^{p, p}(X, \mathbb{R})$ when $n$ tends to infinity. By Poincare duality, it also measures the growth of the norms of $\left(f^{n}\right)_{*}$ acting on $H^{k-p, k-p}(X, \mathbb{R})$. If $X$ is a projective manifold, $d_{p}(f)$ represents the volume growth of $f^{n}(V)$ for $p$-dimensional (closed complex) submanifolds $V$ of $X$. 
It was shown by Sibony and the first author in [6], [7] that dynamical degrees are bi-meromorphic invariants, that is, if $f$ and $g$ are bi-meromorphically conjugate, they have the same dynamical degrees. Dynamical degrees capture important dynamical information, in particular, in the computation of the topological entropy or in the construction of Green currents and of measures of maximal entropy. We refer the reader to the above references and to [8], [10], [15], [20] for more results on this matter.

When $f$ preserves a fibration $\pi: X \rightarrow Y$ as above, the dynamical degree $d_{p}(f \mid \pi)$ of order $p$ of $f$ relative to $\pi$ measures the growth of $\left(f^{n}\right)^{*}$ acting on the subspace $H_{\pi}^{l+p, l+p}(X, \mathbb{R})$ of classes in $H^{l+p, l+p}(X, \mathbb{R})$ which can be supported by a generic fiber of $\pi$. It also measures the growth of $\left(f^{n}\right)_{*}$ acting on $H_{\pi}^{k-p, k-p}(X, \mathbb{R})$ and represents the volume growth of $f^{n}(V)$ for $p$-dimensional submanifolds $V$ of a generic fiber of $\pi$ when $X$ is projective. Precise definitions and properties will be given in Section 3. Here is our main result.

Theorem 1.1. Let $X$ and $Y$ be projective manifolds of dimension $k$ and $l$ respectively with $k \geq l$. Let $f: X \rightarrow X, g: Y \rightarrow Y$ and $\pi: X \rightarrow Y$ be dominant meromorphic maps such that $\pi \circ f=g \circ \pi$. Then the dynamical degrees $d_{p}(f)$ of $f$ are related to the dynamical degrees $d_{p}(g)$ of $g$ and the relative dynamical degrees $d_{p}(f \mid \pi)$ by the formulas

$$
d_{p}(f)=\max _{\max \{0, p-k+l\} \leq j \leq \min \{p, l\}} d_{j}(g) d_{p-j}(f \mid \pi)
$$

for $0 \leq p \leq k$

Note that the condition $\max \{0, p-k+l\} \leq j \leq \min \{p, l\}$ is equivalent to $0 \leq j \leq l$ and $0 \leq p-j \leq k-l$. It guarantees that $d_{j}(g)$ and $d_{p-j}(f \mid \pi)$ are meaningful ${ }^{1}$. We deduce from the above result that $\max d_{p}(f) \geq \max d_{p}(g)$. This gives an affirmative answer to the problem 9.3 in Hasselblatt-Propp [12]. When $X$ and $Y$ have the same dimension, generic fibers of $\pi$ are finite and have the same cardinality. Moreover, $f$ defines bijections between generic fibers of $\pi$. We deduce from the proof of Theorem 1.1 the following corollary which generalizes a result in [6], [7]. It was proved by Nakayama-Zhang for holomorphic maps in [14].

Corollary 1.2. Let $X$ and $Y$ be compact Kähler manifolds of same dimension $k$. Let $f: X \rightarrow X, g: Y \rightarrow Y$ and $\pi: X \rightarrow Y$ be dominant meromorphic maps such that $\pi \circ f=g \circ \pi$. Then the dynamical degrees of $f$ are equal to the dynamical degrees of $g$.

\footnotetext{
${ }^{1}$ We will find later analogous conditions, essentially for the same reason but also to avoid expressions which always vanish, e.g. $\omega_{Y}^{l+1}=0$.
} 
Recall that by a theorem of Khovanskii [13], Teissier [17] and Gromov [9], the dynamical degrees of $f$ are $\log$-concave, i.e. $p \mapsto \log d_{p}(f)$ is concave. Therefore, there are integers $p \leq p^{\prime}$ such that

$$
1=d_{0}(f)<\cdots<d_{p}(f)=\cdots=d_{p^{\prime}}(f)>\cdots>d_{k}(f) .
$$

An instructive example with $p \neq p^{\prime}$ is a map $f\left(x_{1}, x_{2}\right)=\left(h\left(x_{1}\right), x_{2}\right)$ on a product $X_{1} \times X_{2}$ of projective manifolds. A natural problem is to find dynamically interesting examples of maps on projective manifolds. Therefore, it would be interesting to see construction of maps with distinct consecutive dynamical degrees, i.e. with $p=p^{\prime}$. Somehow, this condition insures that there is no trivial direction in the associated dynamical systems. We have the following useful results.

Corollary 1.3. Let $f, \pi, g$ be as in Theorem 1.1. If the consecutive dynamical degrees of $f$ are distinct, then the same property holds for $g$ and for the consecutive dynamical degrees of $f$ relative to $\pi$.

The following result is obtained using the Iitaka fibrations of $X$.

Corollary 1.4. Let $X$ be a projective manifold admitting a dominant meromorphic map with distinct consecutive dynamical degrees. Then the Kodaira dimension of $X$ is either equal to 0 or $-\infty$.

Note that the same result was proved for compact Kähler surfaces by Cantat in [3] and Guedj in [11], and for holomorphic maps on compact Kähler manifolds by Nakayama and Zhang in [14], [21]. We also refer to Amerik-Campana [1] and Nakayama-Zhang [14], [22] for other invariant fibrations for which Theorem 1.1 may be applied in order to compute dynamical degrees.

Acknowledgment. The paper was written while the second author was visiting the Abdus Salam International Centre for Theoretical Physics in Trieste and the Korea Institute for Advanced Study in Seoul. He wishes to express his gratitude to these organizations.

\section{Positive closed currents}

The proof of our main result uses a delicate calculus on positive closed currents on compact Kähler manifolds ${ }^{2}$. In this section, we prove some useful results which can be applied to currents of integration on varieties and may have independent interest. The reader will find in Demailly [4] and Voisin [19] the basic facts on currents and on Kähler geometry.

\footnotetext{
${ }^{2}$ In this paper, we only consider the strong positivity, see e.g. [8], A.2, for the terminology.
} 
Let $\left(X, \omega_{X}\right)$ be a compact Kähler manifold of dimension $k$. Let $\mathcal{K}^{p}(X)$ denote the cone of classes of strictly positive closed $(p, p)$-forms in $H^{p, p}(X, \mathbb{R})$. This is an open cone which is salient, i.e. $\overline{\mathcal{K}^{p}(X)} \cap-\overline{\mathcal{K}^{p}(X)}=\{0\}$. If $c, c^{\prime}$ are two classes in $H^{p, p}(X, \mathbb{R})$, we write $c \leq c^{\prime}$ and $c^{\prime} \geq c$ when $c^{\prime}-c$ is in $\mathcal{K}^{p}(X) \cup\{0\}$.

If $T$ is a real closed $(p, p)$-current, denote by $\{T\}$ its class in $H^{p, p}(X, \mathbb{R})$. If moreover $T$ is positive, the mass of $T$ is defined by $\|T\|:=\left\langle T, \omega_{X}^{k-p}\right\rangle$. We often use the properties that $\|T\|$ depends only on the class of $T$ and $\{T\} \leq A\|T\|\left\{\omega_{X}^{p}\right\}$ for some constant $A>0$ independent of $T$. The following semi-regularization of currents was proved by Sibony and the first author in [6], [7].

Proposition 2.1. Let $T$ be a positive closed ( $p, p)$-current on a compact Kähler manifold $\left(X, \omega_{X}\right)$. Then there is a sequence of smooth positive closed $(p, p)$-forms $T_{n}$ on $X$ which converges weakly to a positive closed $(p, p)$-current $T^{\prime}$ such that $T^{\prime} \geq T$, i.e. $T^{\prime}-T \geq 0,\left\|T_{n}\right\| \leq A\|T\|$ and $\left\{T_{n}\right\} \leq A\|T\|\left\{\omega_{X}^{p}\right\}$, where $A>0$ is a constant independent of $T$. Moreover, if $T$ is smooth on an open set $U$, then for every compact set $K \subset U$, we have $T_{n} \geq T$ on $K$ when $n$ is large enough.

We need the following lemma.

Lemma 2.2. Let $T$ and $S$ be positive closed currents on $X$ of bidegree $(p, p)$ and $(q, q)$ respectively with $p+q \leq k$. Assume that $T$ is smooth on a dense Zariski open set $U$ of $X$. Then $T_{\mid U} \wedge S_{\mid U}$ has a finite mass. More precisely, there is a constant $A>0$ independent of $T, S$ and $U$ such that

$$
\left\|T_{\mid U} \wedge S_{\mid U}\right\|:=\left\langle T_{\mid U} \wedge S_{\mid U}, \omega_{X}^{k-p-q}\right\rangle \leq A\|T\|\|S\| .
$$

Proof. Let $T_{n}$ and $K$ be as in Proposition 2.1. Since $\left\|T_{n} \wedge S\right\|$ can be computed cohomologically, we have

$$
\left\|T_{\mid K} \wedge S_{\mid K}\right\| \leq \liminf _{n \rightarrow \infty}\left\|T_{n} \wedge S\right\| \leq A\|T\|\left\|\omega_{X}^{p} \wedge S\right\|=A\|T\|\|S\| .
$$

This property holds for every compact subset $K$ of $U$. Therefore,

$$
\left\|T_{\mid U} \wedge S_{\mid U}\right\| \leq A\|T\|\|S\| .
$$

The lemma follows.

Consider currents $T$ and $S$ as in Lemma 2.2. So, $T_{\mid U} \wedge S_{\mid U}$ has a finite mass. Therefore, by Skoda's theorem [16], its trivial extension defines a positive closed current on $X$. We denote by $T \stackrel{\circ}{\wedge} S$ this current obtained for the maximal Zariski open set $U$ on which $T$ is smooth (in that case $T_{\mid U}$ is the regular part of $T$ ). Observe that when $S$ has no mass on proper analytic subsets of $X$, the current obtained in this 
Vol. 86 (2011) Comparison of dynamical degrees for semi-conjugate meromorphic maps 821

way does not change if we replace $U$ with another dense Zariski open set. We often use this property in the sequence. By Lemma 2.2, we always have

$$
\|T \stackrel{\circ}{\wedge} S\| \leq A\|T\|\|S\|
$$

We will be interested in positive closed currents $T$ on $Y \times \mathbb{P}^{m}$, where $\left(Y, \omega_{Y}\right)$ is a compact Kähler manifold of dimension $l$ and $\mathbb{P}^{m}$ is the projective space of dimension $m$ endowed with the standard Fubini-Study form $\omega_{\mathrm{FS}}$. We assume that $\omega_{\mathrm{FS}}$ is normalized so that $\omega_{\mathrm{FS}}^{m}$ is a probability measure. In practice, we will take $m:=k-l=\operatorname{dim} X-\operatorname{dim} Y$. In order to simplify the notation, the pull-back of $\omega_{Y}$ and $\omega_{\mathrm{FS}}$ to $Y \times \mathbb{P}^{m}$ under the canonical projections are also denoted by $\omega_{Y}$ and $\omega_{\mathrm{FS}}$. Consider on $Y \times \mathbb{P}^{m}$ the Kähler form $\omega:=\omega_{Y}+\omega_{\mathrm{FS}}$. The pull-back of a class $c$ in $H^{*}(Y, \mathbb{C})$ or $H^{*}\left(\mathbb{P}^{m}, \mathbb{C}\right)$ to $H^{*}\left(Y \times \mathbb{P}^{m}, \mathbb{C}\right)$ under the canonical projections is also denoted by $c$.

If $T$ is a positive closed $(p, p)$-current on $Y \times \mathbb{P}^{m}$, define for $\max \{0, p-m\} \leq$ $j \leq \min \{l, p\}$ (or equivalently, for $0 \leq j \leq l$ and $0 \leq p-j \leq m$ )

$$
\alpha_{j}(T):=\left\langle T, \omega_{Y}^{l-j} \wedge \omega_{\mathrm{FS}}^{m-p+j}\right\rangle .
$$

Observe that $\alpha_{j}(T)$ depends only on the class $\{T\}$ of $T$. Denote by $\cup$ the cup-product on Hodge cohomology groups.

Proposition 2.3. Let $T$ be a positive closed (p,p)-current on $Y \times \mathbb{P}^{m}$ as above. Then

$$
\{T\} \leq A \sum_{\max \{0, p-m\} \leq j \leq \min \{l, p\}} \alpha_{j}(T)\left\{\omega_{Y}^{j}\right\} \cup\left\{\omega_{\mathrm{FS}}^{p-j}\right\},
$$

where $A>0$ is a constant independent of $T$.

Proof. By the Künneth formula (see e.g. [19], p. 266) we have

$$
H^{*}\left(Y \times \mathbb{P}^{m}, \mathbb{C}\right)=H^{*}(Y, \mathbb{C}) \otimes H^{*}\left(\mathbb{P}^{m}, \mathbb{C}\right) .
$$

Therefore, there are classes $c_{j} \in H^{j, j}(Y, \mathbb{R})$ such that

$$
\{T\}=\sum_{\max \{0, p-m\} \leq j \leq \min \{l, p\}} c_{j} \cup\left\{\omega_{\mathrm{FS}}^{p-j}\right\} .
$$

Let $S$ be a smooth positive closed $(l-j, l-j)$-form on $Y$ and $S^{\prime}$ its canonical pull-back to $Y \times \mathbb{P}^{m}$. Recall that $c_{j}$ denotes also the pull-back of $c_{j}$ to $Y \times \mathbb{P}^{m}$. Since $\omega_{\mathrm{FS}}^{m}$ is a probability measure on $\mathbb{P}^{m}$, a simple computation on bidegree gives

$$
c_{j} \cup\{S\}=c_{j} \cup\left\{S^{\prime}\right\} \cup\left\{\omega_{\mathrm{FS}}^{m}\right\}=\left\langle T, S^{\prime} \wedge \omega_{\mathrm{FS}}^{m-p+j}\right\rangle \geq 0 .
$$


So, $c_{j}$ belongs to the convex closed cone $\mathcal{K}$ of classes $c$ in $H^{j, j}(Y, \mathbb{R})$ with $c \cup c^{\prime} \geq 0$ for $c^{\prime} \in \mathcal{K}^{l-j}(Y)$. Since $\mathcal{K}^{l-j}(Y)$ is open and since $\cup$ is non-degenerate, $\mathcal{K}$ is salient, i.e. $\mathcal{K} \cap-\mathcal{K}=\{0\}$. The fact that $\left\{\omega_{Y}^{l-j}\right\}$ is in the interior of $\mathcal{K}^{l-j}(Y)$ implies that $c_{j} \cup\left\{\omega_{Y}^{l-j}\right\}=0$ only when $c_{j}=0$. Moreover, we have

$$
\left\|c_{j}\right\| \leq A^{\prime} c_{j} \cup\left\{\omega_{Y}^{l-j}\right\}=A^{\prime}\left\langle T, \omega_{Y}^{l-j} \wedge \omega_{\mathrm{FS}}^{m-p+j}\right\rangle=A^{\prime} \alpha_{j}(T)
$$

for a fixed norm \|\| on $H^{j, j}(Y, \mathbb{R})$ and for some constant $A^{\prime}>0$. It follows that

$$
c_{j} \leq A \alpha_{j}(T)\left\{\omega_{Y}^{j}\right\}
$$

for some constant $A>0$. The result follows.

Proposition 2.4. Let $T$ be a positive closed ( $p, p)$-current on $Y \times \mathbb{P}^{m}$ as above. Assume that $Y$ is a projective manifold. Then there is a sequence of smooth positive closed ( $p, p)$-forms $T_{n}$ on $Y \times \mathbb{P}^{m}$ which converges weakly to a current $T^{\prime} \geq T$ such that $\alpha_{j}\left(T_{n}\right) \leq A \alpha_{j}(T)$ for all $j$, where $A>0$ is a constant independent of $T$. Moreover, if $T$ is smooth on an open set $U$, then for every compact subset $K$ of $U$ and every $\epsilon>0$, we have $T_{n} \geq T-\epsilon \omega^{p}$ on $K$ when $n$ is large enough.

Proof. We first consider the case where $Y=\mathbb{P}^{l}$ and $\omega_{Y}$ is the Fubini-Study form normalized so that $\omega_{Y}^{l}$ is a probability measure. The Künneth formula applied to this particular case says that $T$ is cohomologous to

$$
\sum_{\max \{0, p-m\} \leq j \leq \min \{l, p\}} \alpha_{j}(T)\left\{\omega_{Y}^{j}\right\} \cup\left\{\omega_{\mathrm{FS}}^{p-j}\right\} .
$$

Since $Y \times \mathbb{P}^{m}$ is homogeneous, we can regularize $T$ using the automorphisms of $Y \times \mathbb{P}^{m}$ which are close to the identity.

More precisely, let $v_{n}$ be a sequence of smooth probability measures on the group of automorphisms Aut $\left(Y \times \mathbb{P}^{m}\right)$ of $Y \times \mathbb{P}^{m}$ whose supports converge to the identity id $\in \operatorname{Aut}\left(Y \times \mathbb{P}^{m}\right)$. Define

$$
T_{n}:=\int_{\tau \in \operatorname{Aut}\left(Y \times \mathbb{P}^{m}\right)} \tau_{*}(T) d v_{n}(\tau) .
$$

Then, the $T_{n}$ are smooth positive closed $(p, p)$-forms and converge weakly to $T$. We also have $\left\{T_{n}\right\}=\{T\}$ and hence $\alpha_{j}\left(T_{n}\right)=\alpha_{j}(T)$. This gives the first assertion for $Y=\mathbb{P}^{l}$.

For the second assertion, we can prove a stronger property. Let $\Phi$ be a smooth positive ( $p, p$ )-form on $U$ such that $\Phi \leq T$. We do not assume that $T$ is smooth nor that $\Phi$ is closed on $U$. Then

$$
\Phi_{n}:=\int_{\tau \in \operatorname{Aut}\left(Y \times \mathbb{P}^{m}\right)} \tau_{*}(\Phi) d v_{n}(\tau)
$$


converges uniformly to $\Phi$ on $K$. Since $\Phi_{n} \leq T_{n}$, we have $T_{n} \geq \Phi-\epsilon \omega^{p}$ on $K$ for $n$ large enough. With our hypothesis, $T$ is smooth on $U$ and we can replace $\Phi$ with $T$.

Assume now that $Y$ is a general projective manifold. We may find a finite family of open holomorphic maps $\Psi_{i}, 1 \leq i \leq s$, from $Y$ onto $\mathbb{P}^{l}$ such that for every point $y \in Y$ at least one map $\Psi_{i}$ is of maximal rank at $y$. To do this it suffices to embed $Y$ into a projective space and take a family of central projections. Let $\Pi_{i}: Y \times \mathbb{P}^{m} \rightarrow \mathbb{P}^{l} \times \mathbb{P}^{m}$ be defined by

$$
\Pi_{i}(y, z):=\left(\Psi_{i}(y), z\right), \quad(y, z) \in Y \times \mathbb{P}^{m} .
$$

We apply the first case to the currents $T^{(i)}:=\left(\Pi_{i}\right)_{*}(T)$.

We construct as above smooth positive closed $(p, p)$-forms $T_{n}^{(i)}$ on $\mathbb{P}^{l} \times \mathbb{P}^{m}$ converging to $T^{(i)}$ such that $\left\{T_{n}^{(i)}\right\}=\left\{T^{(i)}\right\}$. Define $T_{n}:=\sum_{i} \Pi_{i}^{*}\left(T_{n}^{(i)}\right)$. Since the cohomology classes of $T_{n}^{(i)}$ are bounded, the classes of $T_{n}$ are also bounded. Therefore, the masses of $T_{n}$ are bounded. Up to extracting a subsequence, we can assume that $\Pi_{i}^{*}\left(T_{n}^{(i)}\right)$ converges and hence the $T_{n}$ converge to a positive closed current $T^{\prime}$. If $(y, z)$ is a point in $Y \times \mathbb{P}^{m}$ and $\Psi_{i}$ has maximal rank at $y$, then $\Pi_{i}$ defines a local bi-holomorphic map on a neighbourhood of $(y, z)$. In this neighbourhood, we have

$$
T \leq \Pi_{i}^{*}\left(\Pi_{i}\right)_{*}(T)=\Pi_{i}^{*}\left(T^{(i)}\right) \leq \lim _{n \rightarrow \infty} \Pi_{i}^{*}\left(T_{n}^{(i)}\right) \leq T^{\prime} .
$$

The choice of $\Psi_{i}$ implies that $T \leq T^{\prime}$ on $Y \times \mathbb{P}^{m}$. The second assertion of the proposition is a local property. So, it is also easy to check.

It remains to prove the estimate on $\alpha_{j}\left(T_{n}\right)$. Let $\tilde{\omega}_{\mathrm{FS}}$ denote the Fubini-Study form of $\mathbb{P}^{l}$ normalized so that $\tilde{\omega}_{\mathrm{FS}}^{l}$ is a probability measure. Since $\tilde{\omega}_{\mathrm{FS}}$ is strictly positive, there is a constant $A_{1}>0$ such that $\left(\Psi_{i}\right)_{*}\left\{\omega_{Y}^{l-j}\right\} \leq A_{1}\left\{\tilde{\omega}_{\mathrm{FS}}^{l-j}\right\}$. We also have $\left(\Psi_{i}\right)^{*}\left(\tilde{\omega}_{\mathrm{FS}}^{l-j}\right) \leq A_{2} \omega_{Y}^{l-j}$ for some constant $A_{2}>0$. For simplicity, we will also denote by $\omega_{Y}, \omega_{\mathrm{FS}}$ and $\tilde{\omega}_{\mathrm{FS}}$ the pull-backs of these forms to $Y \times \mathbb{P}^{m}$ or to $\mathbb{P}^{l} \times \mathbb{P}^{m}$. In particular, $\left(\Pi_{i}\right)_{*}\left(\omega_{Y}^{l-j} \wedge \omega_{\mathrm{FS}}^{m-p+j}\right)$ and $\left(\Psi_{i}\right)_{*}\left(\omega_{Y}^{l-j}\right) \wedge \omega_{\mathrm{FS}}^{m-p+j}$ represent the same form on $\mathbb{P}^{l} \times \mathbb{P}^{m}$. Since the $T_{n}^{(i)}$ are smooth and since the following integrals can be computed cohomologically, we have

$$
\begin{aligned}
\left\langle\Pi_{i}^{*}\left(T_{n}^{(i)}\right), \omega_{Y}^{l-j} \wedge \omega_{\mathrm{FS}}^{m-p+j}\right\rangle & =\left\langle T_{n}^{(i)},\left(\Psi_{i}\right)_{*}\left(\omega_{Y}^{l-j}\right) \wedge \omega_{\mathrm{FS}}^{m-p+j}\right\rangle \\
& \leq A_{1}\left\langle T_{n}^{(i)}, \tilde{\omega}_{\mathrm{FS}}^{l-j} \wedge \omega_{\mathrm{FS}}^{m-p+j}\right\rangle \\
& =A_{1}\left\langle T^{(i)}, \tilde{\omega}_{\mathrm{FS}}^{l-j} \wedge \omega_{\mathrm{FS}}^{m-p+j}\right\rangle \\
& =A_{1}\left\langle T, \Psi_{i}^{*}\left(\tilde{\omega}_{\mathrm{FS}}^{l-j}\right) \wedge \omega_{\mathrm{FS}}^{m-p+j}\right\rangle \\
& \leq A_{1} A_{2}\left\langle T, \omega_{Y}^{l-j} \wedge \omega_{\mathrm{FS}}^{m-p+j}\right\rangle .
\end{aligned}
$$

It follows that $\alpha_{j}\left(\Pi_{i}^{*}\left(T_{n}^{(i)}\right)\right) \leq A_{1} A_{2} \alpha_{j}(T)$ and hence $\alpha_{j}\left(T_{n}\right) \leq A \alpha_{j}(T)$ for some constant $A>0$. 


\section{Dynamical degrees}

Let $\pi:\left(X, \omega_{X}\right) \rightarrow\left(Y, \omega_{Y}\right)$ be a dominant meromorphic map between compact Kähler manifolds of dimension $k$ and $l$ respectively. The map $\pi$ is holomorphic outside the indeterminacy set $I_{\pi}$ which is an analytic subset of $X$ of codimension at least 2. The closure $\Gamma$ of its graph over $X \backslash I_{\pi}$ is an irreducible analytic subset of dimension $k$ of $X \times Y$. If, $\tau_{X}$ and $\tau_{Y}$ denote the projections from $X \times Y$ onto its factors, then $\tau_{X}$ defines a bi-holomorphic map between $\Gamma \backslash \tau_{X}^{-1}\left(I_{\pi}\right)$ and $X \backslash I_{\pi}$. The fibers of $\tau_{X \mid \Gamma}$ over $I_{\pi}$ have positive dimension. One can identify $\pi$ with $\tau_{Y} \circ\left(\tau_{X \mid \Gamma}\right)^{-1}$. For $A \subset$ $X$ and $B \subset Y$, define $\pi(A):=\tau_{Y}\left(\tau_{X \mid \Gamma}\right)^{-1}(A)$ and $\pi^{-1}(B):=\tau_{X}\left(\tau_{Y \mid \Gamma}\right)^{-1}(B)$.

The map $\pi$ induces linear operators on currents. If $\Phi$ is a smooth $(p, q)$-form on $Y$, then $\pi^{*}(\Phi)$ is the $(p, q)$-current defined by

$$
\pi^{*}(\Phi):=\left(\tau_{X}\right)_{*}\left(\tau_{Y}^{*}(\Phi) \wedge[\Gamma]\right),
$$

where $[\Gamma]$ is the current of integration on $\Gamma$. It is not difficult to see that $\pi^{*}(\Phi)$ is an $L^{1}$ form smooth outside $I_{\pi}$. If $\Psi$ is a smooth $(p, q)$-form on $X$ with $p, q \geq k-l$, then $\pi_{*}(\Psi)$ is the $(p-k+l, q-k+l)$-current defined by

$$
\pi_{*}(\Psi):=\left(\tau_{Y}\right)_{*}\left(\tau_{X}^{*}(\Psi) \wedge[\Gamma]\right) .
$$

If $\Phi$ and $\Psi$ are closed or positive, so are $\pi^{*}(\Phi)$ and $\pi_{*}(\Psi)$. Therefore, $\pi^{*}$ and $\pi_{*}$ induce linear operators on the Hodge cohomology groups of $X$ and $Y$.

In general, the above operators do not extend continuously to positive closed currents. We will use instead the strict transforms of currents $\pi^{\bullet}$ and $\pi_{\bullet}$ which coincide with $\pi^{*}$ and $\pi_{*}$ on smooth positive closed forms. In this paper, we only need these operators in the case where $X$ and $Y$ have the same dimension $k$.

Let $U$ be the maximal Zariski open set in $X \backslash I_{\pi}$ such that $\pi: U \rightarrow \pi(U)$ is locally invertible. The complement of $U$ in $X$ is called the critical set of $\pi$. If $T$ is a positive closed $(p, p)$-current on $Y,\left(\pi_{\mid U}\right)^{*}(T)$ is well-defined and is a positive closed $(p, p)$-current on $U$. Proposition 2.1 allows us to show that this current has finite mass. By Skoda's Theorem [16], its trivial extension to $X$ is a positive closed $(p, p)$-current that we denote by $\pi^{\bullet}(T)$.

Let $V$ be the maximal Zariski open set in $Y \backslash \pi\left(I_{\pi}\right)$ such that $\pi: \pi^{-1}(V) \rightarrow V$ is a non-ramified covering. The complement of $V$ in $Y$ is called the set of critical values of $\pi$. If $S$ is a positive closed $(p, p)$-current on $X$, then $\pi_{\bullet}(S)$ is the trivial extension of $\left(\pi_{\mid \pi^{-1}(V)}\right)_{*}(S)$ to $Y$. This is also a positive closed $(p, p)$-current. We will use the properties that $\left\|\pi^{\bullet}(T)\right\| \leq A\|T\|$ and $\left\|\pi_{\bullet}(S)\right\| \leq A\|S\|$ for some constant $A>0$ independent of $T, S$, see [6], [7] for details.

Consider now a dominant meromorphic self-map $f: X \rightarrow X$. The iterate of order $n$ of $f$ is defined by $f^{n}:=f \circ \cdots \circ f$ ( $n$ times) on a dense Zariski open set and extends to a dominant meromorphic map on $X$. Define for $0 \leq p \leq k$

$$
\lambda_{p}\left(f^{n}\right):=\left\|\left(f^{n}\right)^{*}\left(\omega_{X}^{p}\right)\right\|=\left\langle\left(f^{n}\right)^{*}\left(\omega_{X}^{p}\right), \omega_{X}^{k-p}\right\rangle .
$$


Vol. 86 (2011) Comparison of dynamical degrees for semi-conjugate meromorphic maps 825

It is not difficult to see that

$$
\lambda_{p}\left(f^{n}\right)=\left\|\left(f^{n}\right)_{*}\left(\omega_{X}^{k-p}\right)\right\|=\left\langle\left(f^{n}\right)_{*}\left(\omega_{X}^{k-p}\right), \omega_{X}^{p}\right\rangle .
$$

It was shown in [6], [7] that $\left[\lambda_{p}\left(f^{n}\right)\right]^{1 / n}$ converges to a constant $d_{p}(f)$ which is the dynamical degree of order $p$ of $f$. Note that the main difficulty here is that in general we do not have $\left(f^{n+s}\right)^{*}=\left(f^{n}\right)^{*} \circ\left(f^{s}\right)^{*}$ on cohomology classes.

Let \|\|$_{H^{p, p}}$ denote the norm of an operator acting on $H^{p, p}(X, \mathbb{R})$ with respect to a fixed norm on that space. Since the mass of a positive closed current depends only on its cohomology class, we deduce from the above discussion that

$$
A^{-1} \lambda_{p}\left(f^{n}\right) \leq\left\|\left(f^{n}\right)^{*}\right\|_{H^{p, p}} \leq A \lambda_{p}\left(f^{n}\right),
$$

for some constant $A>0$. It follows that

$$
d_{p}(f)=\lim _{n \rightarrow \infty}\left\|\left(f^{n}\right)^{*}\right\|_{H^{p, p}}^{1 / n}
$$

Note that we also have $d_{p}\left(f^{n}\right)=d_{p}(f)^{n}$ for $n \geq 1$. The last dynamical degree $d_{k}(f)$ is also called the topological degree of $f$. It is equal to the number of points in a generic fiber of $f$ and we have $\lambda_{k}\left(f^{n}\right)\left\|\omega_{X}^{k}\right\|^{-1}=d_{k}\left(f^{n}\right)=d_{k}(f)^{n}$.

Proposition 3.1. Let $T$ be a positive closed $(p, p)$-current and $S$ a positive closed $(k-p, k-p)$-current on $X$. Then

$$
\left\|\left(f^{n}\right)^{\bullet}(T)\right\| \leq A\|T\| \lambda_{p}\left(f^{n}\right) \quad \text { and } \quad\left\|\left(f^{n}\right) \bullet(S)\right\| \leq A\|S\| \lambda_{p}\left(f^{n}\right)
$$

for some constant $A>0$ independent of $T, S$ and $n$. In particular, we have

$$
\limsup _{n \rightarrow \infty}\left\|\left(f^{n}\right)^{\bullet}(T)\right\|^{1 / n} \leq d_{p}(f) \text { and } \limsup _{n \rightarrow \infty}\left\|\left(f^{n}\right) \bullet(S)\right\|^{1 / n} \leq d_{p}(f) .
$$

Proof. We show the first inequality. The second one is proved in the same way. Let $T_{i}$ be smooth positive closed forms as in Proposition 2.1. It follows from the definition of $\left(f^{n}\right)^{\bullet}$ that any limit value of $\left(f^{n}\right)^{*}\left(T_{i}\right)$ is larger than or equal to $\left(f^{n}\right)^{\bullet}(T)$. So, it is enough to bound the mass of $\left(f^{n}\right)^{*}\left(T_{i}\right)$. Since this mass can be computed cohomologically and since $\left\{T_{i}\right\} \leq A\|T\|\left\{\omega_{X}^{p}\right\}$, we obtain that $\left\|\left(f^{n}\right)^{*}\left(T_{i}\right)\right\| \leq A\|T\| \lambda_{p}\left(f^{n}\right)$ for some constant $A>0$. This completes the proof.

The above proposition can be applied to currents of integration on submanifolds $V$ of dimension $k-p$ or $p$ of $X$ and gives a upper bound for the volume growth of the preimage or image of $V$ by $f^{n}$.

It was shown in [6], [7] that dynamical degrees are bi-meromorphic invariants, i.e. conjugate maps have the same dynamical degrees. This property allows us to define 
dynamical degrees for maps on singular manifolds having a kählerian desingularization. We will use the same argument in order to define dynamical degrees relative to an invariant meromorphic fibration.

Let $f, g, \pi$ be as in Theorem 1.1. So, $\pi$ defines a fibration and $f$ preserves this fibration. Let us assume first that $\pi$ is a holomorphic map. By the Bertini-Sard theorem, the set $Z$ of critical values of $\pi$ is a proper analytic subset of $Y$. Therefore, $\pi: X \backslash \pi^{-1}(Z) \rightarrow Y \backslash Z$ defines a regular holomorphic fibration. Its fibers form a continuous family of smooth submanifolds of dimension $k-l$ of $X$.

Let $P_{f}$ and $P_{g}$ denote the union of the critical set and the set of critical values of $f$ and $g$ respectively. They contain the indeterminacy sets of $f$ and of $g$. A fiber $L_{y}:=\pi^{-1}(y)$ with $y \in Y \backslash Z$ is called generic if for every $n \geq 0$

(a) $g^{n}(y)$ and $g^{-n}(y)$ do not intersect $P_{g}$;

(b) For every point $b$ in $g^{n}(y) \cup g^{-n}(y)$, no component of $L_{b}$ is contained in $P_{f}$. Denote by $\Sigma$ the set of $y$ such that $L_{y}$ is generic. Observe that $Y \backslash \Sigma$ is contained in a finite or countable union of proper analytic subsets of $Y$. So, $\Sigma$ is connected. We also have $g(\Sigma)=g^{-1}(\Sigma)=\Sigma$. We will use the following lemma for $v=\omega_{Y}^{l}$ and for $v=\left[d_{l}(g)\right]^{-n}\left(g^{n}\right)^{*}\left(\omega_{Y}^{l}\right)$.

Lemma 3.2. Let $L_{y}$ be a generic fiber as above. Let $v$ be a probability measure on $Y$ which has no mass on proper analytic subsets of $Y$. Then, for $0 \leq p \leq k-l$ and for $n \geq 0$, the 6 positive closed currents

$$
d_{l}(g)^{-n}\left(f^{n}\right)^{\bullet}\left(\omega_{X}^{p} \wedge\left[L_{y}\right]\right), \quad\left(f^{n}\right)^{*}\left(\omega_{X}^{p}\right) \stackrel{\circ}{\wedge}\left[L_{y}\right], \quad\left(f^{n}\right)^{*}\left(\omega_{X}^{p}\right) \stackrel{\circ}{\wedge} \pi^{*}(v)
$$

and

$$
\begin{gathered}
\left(f^{n}\right)_{\bullet}\left(\omega_{X}^{k-l-p} \wedge\left[L_{y}\right]\right), \quad d_{l}(g)^{-n}\left(f^{n}\right)_{*}\left(\omega_{X}^{k-l-p}\right) \stackrel{\circ}{\wedge}\left[L_{y}\right], \\
d_{l}(g)^{-l}\left(f^{n}\right)_{*}\left(\omega_{X}^{k-l-p}\right) \stackrel{\circ}{\wedge} \pi^{*}(v)
\end{gathered}
$$

have the same mass. In particular, their mass does not depend on $y \in \Sigma$.

Proof. For $y \in \Sigma$, define

$$
\varphi(y):=d_{l}(g)^{-n}\left\|\left(f^{n}\right)^{\bullet}\left(\omega_{X}^{p} \wedge\left[L_{y}\right]\right)\right\| \quad \text { and } \quad \psi(y):=\left\|\left(f^{n}\right) \bullet\left(\omega_{X}^{k-l-p} \wedge\left[L_{y}\right]\right)\right\| .
$$

It is not difficult to see that these functions are continuous on $\Sigma$. We have

It follows that

$$
\begin{aligned}
\varphi(y) & =d_{l}(g)^{-n}\left\langle\left(f^{n}\right)^{\bullet}\left(\omega_{X}^{p} \wedge\left[L_{y}\right]\right), \omega_{X}^{k-l-p}\right\rangle \\
& =d_{l}(g)^{-n}\left\langle\omega_{X}^{p},\left[L_{y}\right] \stackrel{\circ}{\wedge}\left(f^{n}\right)_{*}\left(\omega_{X}^{k-l-p}\right)\right\rangle .
\end{aligned}
$$

$$
\varphi=d_{l}(g)^{-n} \pi_{*}\left(\omega_{X}^{p} \wedge\left(f^{n}\right)_{*}\left(\omega_{X}^{k-l-p}\right)\right)
$$


Vol. 86 (2011) Comparison of dynamical degrees for semi-conjugate meromorphic maps 827

in the sense of currents on $Y$. Therefore, $\varphi$ defines a closed 0 -current on $Y$ and it should be constant on $\Sigma$.

We also deduce from the above computation that

$$
\varphi(y)=d_{l}(g)^{-n}\left\|\left(f^{n}\right)_{*}\left(\omega_{X}^{k-l-p}\right) \stackrel{\circ}{\wedge}\left[L_{y}\right]\right\| .
$$

Since $v$ has no mass on $Y \backslash \Sigma$, we obtain

$$
\varphi=\int \varphi(y) d v=d_{l}(g)^{-n}\left\|\left(f^{n}\right)_{*}\left(\omega_{X}^{k-l-p}\right) \stackrel{\circ}{\wedge} \pi^{*}(v)\right\| .
$$

In the same way, we prove that $\psi$ is constant on $\Sigma$ and

$$
\psi=\left\|\left(f^{n}\right)^{*}\left(\omega_{X}^{p}\right) \stackrel{\circ}{\wedge}\left[L_{y}\right]\right\|=\left\|\left(f^{n}\right)^{*}\left(\omega_{X}^{p}\right) \stackrel{\circ}{\wedge} \pi^{*}(v)\right\| .
$$

It remains to check that $\varphi=\psi$. Using that $\psi$ is constant and $\# g^{-n}(y)=d_{l}(g)^{n}$, we have

$$
\varphi=d_{l}(g)^{-n}\left\|\left(f^{n}\right)^{\bullet}\left(\omega_{X}^{p} \wedge\left[L_{y}\right]\right)\right\|=d_{l}(g)^{-n} \sum_{b \in g^{-n}(y)}\left\|\left(f^{n}\right)^{*}\left(\omega_{X}^{p}\right) \stackrel{\circ}{\wedge}\left[L_{b}\right]\right\|=\psi .
$$

This completes the proof.

Define $\lambda_{p}\left(f^{n} \mid \pi\right)$ the mass of the currents in Lemma 3.2. We have in particular

$$
\lambda_{p}\left(f^{n} \mid \pi\right)=\left\|\left(f^{n}\right)^{*}\left(\omega_{X}^{p}\right) \wedge \pi^{*}\left(\omega_{Y}^{l}\right)\right\|
$$

Recall that $\pi$ is holomorphic and then $\pi^{*}\left(\omega_{Y}^{l}\right)$ is smooth.

Proposition 3.3. The sequence $\lambda_{p}\left(f^{n} \mid \pi\right)^{1 / n}$ converges to a constant $d_{p}(f \mid \pi)$. Let $T$ be a positive closed $(p+l, p+l)$-current and $S$ a positive closed $(k-p, k-p)$ current on $X$ which are supported on a generic fiber $L_{y}$. Then

$$
\left\|\left(f^{n}\right)^{\bullet}(T)\right\| \leq A_{y}\|T\| d_{l}(g)^{n} \lambda_{p}\left(f^{n} \mid \pi\right) \quad \text { and } \quad\left\|\left(f^{n}\right) \bullet(S)\right\| \leq A_{y}\|S\| \lambda_{p}\left(f^{n} \mid \pi\right)
$$

for some constant $A_{y}>0$ independent of $T$ and $S$. In particular, we have

$$
\limsup _{n \rightarrow \infty}\left\|\left(f^{n}\right)^{\bullet}(T)\right\|^{1 / n} \leq d_{l}(g) d_{p}(f \mid \pi)
$$

and

$$
\limsup _{n \rightarrow \infty}\left\|\left(f^{n}\right) \bullet(S)\right\|^{1 / n} \leq d_{p}(f \mid \pi) .
$$


Proof. Fix a generic fiber $L_{y}$ with $y \in \Sigma$. We will show that

$$
\lambda_{p}\left(f^{n+m} \mid \pi\right) \leq A_{y} \lambda_{p}\left(f^{n} \mid \pi\right) \lambda_{p}\left(f^{m} \mid \pi\right)
$$

for some constant $A_{y}>0$ and for all $n, m \geq 0$. This will imply the first assertion because the sequence $A_{y} \lambda_{p}\left(f^{n} \mid \pi\right)$ is sub-multiplicative.

Since $L_{y}$ is a compact Kähler manifold, we can apply Proposition 2.1 to $L_{y}$. Let $b$ be a point in $\Sigma$ such that $g^{m}(b)=y$. Define $R:=\left(f^{m}\right) \bullet\left(\omega_{X}^{k-l-p} \wedge\left[L_{b}\right]\right)$. This is a positive closed $(k-p, k-p)$-current on $X$ which is also a $(k-l-p, k-l-p)$ current on $L_{y}$. By Lemma 3.2, we have $\|R\|=\lambda_{p}\left(f^{m} \mid \pi\right)$. Therefore, there are smooth positive closed $(k-l-p, k-l-p)$-forms $\Theta_{i}$ on $L_{y}$ which converge to a current $\Theta \geq R$. Moreover, we have $\left\{\Theta_{i}\right\} \leq A_{y} \lambda_{p}\left(f^{m} \mid \pi\right)\left\{\omega_{X \mid L_{y}}^{k-l-p}\right\}$ for some constant $A_{y}>0$, where the inequality is considered in $H^{*}\left(L_{y}, \mathbb{R}\right)$.

Let $h$ denote the restriction of $f^{n}$ to $L_{y}$. It defines a meromorphic map from $L_{y}$ to $L_{g^{n}(y)}$. Since the mass of a positive closed current can be computed cohomologically, we obtain

$$
\begin{aligned}
\lambda_{p}\left(f^{n+m} \mid \pi\right) & =\left\|\left(f^{n}\right) \bullet(R)\right\| \leq \liminf _{i \rightarrow \infty}\left\|h_{*}\left(\Theta_{i}\right)\right\| \leq A_{y} \lambda_{p}\left(f^{m} \mid \pi\right)\left\|h_{*}\left(\omega_{X \mid L_{y}}^{k-l-p}\right)\right\| \\
& =A_{y} \lambda_{p}\left(f^{m} \mid \pi\right)\left\|\left(f^{n}\right) \bullet\left(\omega_{X}^{k-l-p} \wedge\left[L_{y}\right]\right)\right\| \\
& =A_{y} \lambda_{p}\left(f^{m} \mid \pi\right) \lambda_{p}\left(f^{n} \mid \pi\right) .
\end{aligned}
$$

This implies the first assertion in the proposition. The rest is proved in the same way using the semi-regularization result for $T$ and $S$ on $L_{y}$, see also Proposition 3.1.

We call $d_{p}(f \mid \pi)$ the dynamical degree of order $p$ of $f$ relative to $\pi$. The convergence in Proposition 3.3 implies that $d_{p}\left(f^{n} \mid \pi\right)=d_{p}(f \mid \pi)^{n}$.

Remark 3.4. Our choice of $\Sigma$ simplifies the calculus on currents but several properties above still hold for some $y \notin \Sigma$. For example, if $y$ is a fixed point of $g$ which is not a critical value of $\pi$ and if no component of $L_{y}$ is contained in the critical set of $f$, then $d_{p}(f \mid \pi)=d_{p}\left(f_{\mid L_{y}}\right)$. The proof is left to the reader.

The next result shows that the relative dynamical degrees are bi-meromorphic invariants. Consider a bi-meromorphic map $\tau:\left(\tilde{X}, \omega_{\tilde{X}}\right) \rightarrow\left(X, \omega_{X}\right)$ between compact Kähler manifolds. Define $\tilde{\pi}:=\pi \circ \tau$ and $\tilde{f}:=\tau^{-1} \circ f \circ \tau$. Then, $\tilde{f}$ is a dominant meromorphic map conjugate to $f$ and $\tilde{\pi} \circ \tilde{f}=g \circ \tilde{\pi}$.

Proposition 3.5. Assume that $\tilde{\pi}$ is holomorphic. Then

$$
d_{p}(f \mid \pi)=d_{p}(\tilde{f} \mid \tilde{\pi})
$$

for $0 \leq p \leq k-l$. 
Vol. 86 (2011) Comparison of dynamical degrees for semi-conjugate meromorphic maps 829

Proof. Since $\tau$ is bi-meromorphic, $\tau_{\bullet} \tilde{\pi}^{*}\left(\omega_{Y}^{l}\right)=\pi^{*}\left(\omega_{Y}^{l}\right)$ and $\tilde{f}^{n}=\tau^{-1} \circ f^{n} \circ \tau$, we have

$$
\begin{aligned}
\lambda_{p}\left(\tilde{f}^{n} \mid \tilde{\pi}\right) & =\left\langle\left(\tilde{f}^{n}\right)^{*}\left(\omega_{\tilde{X}}^{p}\right) \wedge \tilde{\pi}^{*}\left(\omega_{Y}^{l}\right), \omega_{\tilde{X}}^{k-l-p}\right\rangle \\
& =\left\langle\tau^{\bullet}\left(f^{n}\right)^{\bullet} \tau_{*}\left(\omega_{\tilde{X}}^{p}\right) \wedge \omega_{\tilde{X}}^{k-l-p}, \tilde{\pi}^{*}\left(\omega_{Y}^{l}\right)\right\rangle \\
& =\left\langle\left(f^{n}\right)^{\bullet} \tau_{*}\left(\omega_{\tilde{X}}^{p}\right) \stackrel{\circ}{\wedge} \tau_{*}\left(\omega_{\tilde{X}}^{k-l-p}\right), \pi^{*}\left(\omega_{Y}^{l}\right)\right\rangle .
\end{aligned}
$$

Using the semi-regularization result for $\tau_{*}\left(\omega_{\widetilde{X}}^{k-l-p}\right)$, we deduce that

$$
\lambda_{p}\left(\tilde{f}^{n} \mid \tilde{\pi}\right) \leq A\left\langle\left(f^{n}\right)^{\bullet} \tau_{*}\left(\omega_{\tilde{X}}^{p}\right) \wedge \omega_{X}^{k-l-p}, \pi^{*}\left(\omega_{Y}^{l}\right)\right\rangle
$$

for some constant $A>0$. Then, using a semi-regularization of $\tau_{*}\left(\omega_{\tilde{X}}^{p}\right)$, we obtain

$$
\lambda_{p}\left(\tilde{f}^{n} \mid \tilde{\pi}\right) \leq A^{\prime}\left\langle\left(f^{n}\right)^{*}\left(\omega_{X}^{p}\right) \wedge \omega_{X}^{k-l-p}, \pi^{*}\left(\omega_{Y}^{l}\right)\right\rangle=A^{\prime} \lambda_{p}\left(f^{n} \mid \pi\right)
$$

for some constant $A^{\prime}>0$. It follows that $d_{p}(\tilde{f} \mid \tilde{\pi}) \leq d_{p}(f \mid \pi)$. The converse inequality is proved in the same way.

The last proposition allows us to define relative dynamical degrees in the general case. Assume now that $f$ preserves a meromorphic fibration $\pi: X \rightarrow Y$, i.e. $\pi \circ f=$ $g \circ \pi$ as in Theorem 1.1. Let $\Gamma$ denote the closure of the graph of $\pi$ in $X \times Y$. Then $\Gamma$ is an irreducible analytic set of dimension $k$ which is bi-meromorphic to $X$. Let $\sigma: \tilde{X} \rightarrow \Gamma$ be a desingularization of $\Gamma$ which can be constructed using a blow-up along the singularities. By Blanchard's theorem [2], $\tilde{X}$ is a compact Kähler manifold. Then, $\tau:=\tau_{X} \circ \sigma$ is a bi-meromorphic map from $\tilde{X}$ to $X$. Define also $\tilde{\pi}:=\tau_{Y} \circ \sigma$ and $\tilde{f}:=\tau^{-1} \circ f \circ \tau$. The map $\tilde{\pi}$ is holomorphic and $\tilde{\pi} \circ \tilde{f}=g \circ \tilde{\pi}$. Define the dynamical degree of order $p$ of $f$ relative to $\pi$ by

$$
d_{p}(f \mid \pi):=d_{p}(\tilde{f} \mid \tilde{\pi}) .
$$

Proposition 3.5 implies that the definition does not depend on the choice of $\sigma$. The following result is a consequence of a theorem by Khovanskii, Teissier and Gromov.

Proposition 3.6. The function $p \mapsto \log d_{p}(f \mid \pi)$ is concave for $0 \leq p \leq k-l$. In particular, $d_{p}(f \mid \pi) \geq 1$ for $0 \leq p \leq k-l$.

Proof. We can assume that $\pi$ is holomorphic. We have to show that

$$
d_{p-1}(f \mid \pi) d_{p+1}(f \mid \pi) \leq d_{p}(f \mid \pi)^{2} .
$$

For this purpose, it is enough to check that

$$
\lambda_{p-1}\left(f^{n} \mid \pi\right) \lambda_{p+1}\left(f^{n} \mid \pi\right) \leq \lambda_{p}\left(f^{n} \mid \pi\right)^{2} .
$$


Observe that for non-critical values $y$ of $\pi$, the fibers $L_{y}$ are not necessarily connected but they contain the same number $s$ of components. The family of these components is connected since $X$ is connected. It defines a covering of degree $s$ over the set of non-critical values of $\pi$. Let $\Sigma^{\prime}$ denote the parameter space for the components $L_{y}^{\prime}$ of $L_{y}$ with $y \in \Sigma$. We may think of $\Sigma^{\prime}$ as a covering of degree $s$ over $\Sigma$. We can then prove as in Lemma 3.2 that the function $L_{y}^{\prime} \mapsto\left\|\left(f^{n}\right)^{*}\left(\omega_{X}^{p}\right) \stackrel{\circ}{\wedge}\left[L_{y}^{\prime}\right]\right\|$ is constant on $\Sigma^{\prime}$. Therefore, it is equal to $s^{-1}\left\|\left(f^{n}\right)^{*}\left(\omega_{X}^{p}\right) \stackrel{\circ}{\wedge}\left[L_{y}\right]\right\|$ and then to $s^{-1} \lambda_{p}\left(f^{n} \mid \pi\right)$.

Let $h$ be the restriction of $f^{n}$ to $L_{1}:=L_{y}^{\prime}$ and define $L_{2}:=h\left(L_{1}\right)$. Let $\Gamma$ denote the graph of $h$ in $L_{1} \times L_{2}$ and $\tau: \hat{\Gamma} \rightarrow \Gamma$ a desingularization of $\Gamma$ using some blow-up along the singularities. By Blanchard's theorem [2], $\widetilde{\Gamma}$ is a compact Kähler manifold. Denote by $\tau_{1}: \hat{\Gamma} \rightarrow L_{1}$ and $\tau_{2}: \hat{\Gamma} \rightarrow L_{2}$ the canonical projections. We have $h=\tau_{2} \circ \tau_{1}^{-1}$. Define $\omega_{1}:=\tau_{1}^{*}\left(\omega_{X}\right)$ and $\omega_{2}:=\tau_{2}^{*}\left(\omega_{X}\right)$. We deduce from the above discussion that

$$
s^{-1} \lambda_{p}\left(f^{n} \mid \pi\right)=\left\|\left(h^{n}\right)^{*}\left(\omega_{X}^{p}\right)\right\|=\int_{\widehat{\Gamma}} \omega_{1}^{k-l-p} \wedge \omega_{2}^{p} .
$$

If $\gamma_{p}$ denotes the last integral, Gromov proved in [9] that $p \mapsto \log \gamma_{p}$ is concave, i.e. $\gamma_{p-1} \gamma_{p+1} \leq \gamma_{p}^{2}$, when $\omega_{1}$ and $\omega_{2}$ are Kähler forms. By continuity, this still holds in our case where these forms are only smooth positive and closed. Hence, $p \mapsto \log d_{p}(f \mid \pi)$ is concave.

In order to deduce the second assertion of the proposition, it is enough to show that $d_{0}(f \mid \pi)=1$ and $d_{k-l}(f \mid \pi) \geq 1$. For $y$ generic, we have

$$
\lambda_{0}\left(f^{n} \mid \pi\right)=d_{l}(g)^{-n}\left\|\left(f^{n}\right)^{\bullet}\left[L_{y}\right]\right\|=d_{l}(g)^{-n} \sum_{b \in g^{-n}(y)}\left\|\left[L_{b}\right]\right\| .
$$

Hence, $\lambda_{0}\left(f^{n} \mid \pi\right)$ is independent of $n$ since $\# g^{-n}(y)=d_{l}(g)^{n}$ and the mass of $\left[L_{b}\right]$, with $b \in \Sigma$, is independent of $b$. It follows that $d_{0}(f \mid \pi)=1$.

We also have for $y$ generic and $b \in g^{-n}(y)$

$$
\lambda_{k-l}\left(f^{n} \mid \pi\right)=\left\|\left(f^{n}\right) \bullet\left[L_{b}\right]\right\| \geq\left\|\left[L_{y}\right]\right\| .
$$

So, the sequence $\lambda_{k-l}\left(f^{n} \mid \pi\right)$ is bounded from below by a positive constant. Therefore, $d_{k-l}(f \mid \pi) \geq 1$. This completes the proof of the lemma. Note that we can show that $d_{k-l}(f \mid \pi)$ is the number of points in a generic fiber of the restriction of $f$ to $L_{y}$.

Consider now some examples, see also [1], [14], [21], [22].

Example 3.7. Let $X=Y \times Z$ be the product of two compact Kähler manifolds and $\pi: X \rightarrow Y$ the canonical projection. Consider $f(y, z):=(g(y), h(z))$ where 
$g: Y \rightarrow Y$ and $h: Z \rightarrow Z$ are dominant meromorphic maps. So, $f$ is semiconjugate to $g$. The relative dynamical degree $d_{p}(f \mid \pi)$ is equal to $d_{p}(h)$. We easily deduce from the definition of dynamical degrees that

$$
d_{p}(f)=\max _{\max \{0, p-k+l\} \leq j \leq \min \{p, l\}} d_{j}(g) d_{p-j}(h) .
$$

There are more interesting examples of maps on the product $Y \times Z$. Let $F$ be a compact Kähler manifold. Assume that $F$ is also the parameter space of a meromorphic family of meromorphic self-maps of $Z$. Let $\tau: Y \rightarrow F$ be a meromorphic map. Then $f(y, z):=(g(y), \tau(y)(z))$ is a meromorphic self-map of $Y \times Z$ which preserves the fibration $\pi$. The example is also interesting when $\tau(y)$ is holomorphic for generic $y$ or when a Zariski open set $G$ of $F$ is a Lie group and $\tau$ is a morphism from $G$ to the group of bi-meromorphic maps of $Z$.

Example 3.8. Let $g: Y \rightarrow Y$ be a dominant meromorphic map on a compact Kähler manifold $Y$. It induces a meromorphic self-map $f$ on the projectivization $X:=\mathbb{P} T_{Y}$ of the holomorphic tangent bundle of $Y$. The map $f$ preserves the fibration associated to the canonical projection from $X$ onto $Y$ and is semi-conjugate to $g$. This example and some applications were considered in [5].

\section{Proofs of the results}

We first prove Theorem 1.1. Since the dynamical degrees are bi-meromorphic invariants, Proposition 3.5 allows us to assume that $\pi$ is a holomorphic map. Since $X$ is projective, we can construct a dominant meromorphic map $v: X \rightarrow \mathbb{P}^{k-l}$. Indeed, it is enough to embed $X$ in a projective space and choose a generic central projection on $\mathbb{P}^{k-l}$. Replacing $X$ with a desingularization of the graph of $v$ allows to assume that $v$ is holomorphic. Consider the holomorphic map $\Pi: X \rightarrow Y \times \mathbb{P}^{k-l}$ defined by

$$
\Pi(x):=(\pi(x), v(x)) .
$$

Since the chosen central projection is generic, the intersection of a generic fiber of $\pi$ and a generic fiber of $v$ is finite. Therefore, $\Pi$ is dominant.

Our proof is based on a delicate calculus on currents. If $X=Y \times \mathbb{P}^{k-l}$ and $\pi$ is the canonical projection onto $Y$, the proof is simpler and the properties obtained in Section 2 can be directly applied. A rough idea is to reduce the general case to the particular case using the map $\Pi$. In other words, we use the fact that $f$ is, in some sense, "semi-conjugate" to the multi-valued map $\Pi \circ f \circ \Pi^{-1}$ which is defined on $Y \times \mathbb{P}^{k-l}$.

Let $\omega_{\mathrm{FS}}$ denote the Fubini-Study form on $\mathbb{P}^{k-l}$. For simplicity, the canonical pull-back of $\omega_{Y}$ and $\omega_{\mathrm{FS}}$ to $Y \times \mathbb{P}^{k-l}$ are still denoted by $\omega_{Y}$ and $\omega_{\mathrm{FS}}$. In particular, 
$\Pi^{*}\left(\omega_{Y}\right)$ and $\pi^{*}\left(\omega_{Y}\right)$ represent the same form on $X$. We consider on $Y \times \mathbb{P}^{k-l}$ the Kähler form $\omega:=\omega_{Y}+\omega_{\mathrm{FS}}$. Our calculus will involve the quantities $a_{q, p}(n)$ defined for $n \geq 0,0 \leq q \leq k-l$ and $q \leq p \leq l+q$ by

$$
\begin{aligned}
a_{q, p}(n): & =\left\|\Pi_{*}\left(f^{n}\right)^{*} \Pi^{*}\left(\omega^{p}\right) \wedge \omega_{Y}^{l-p+q}\right\| \\
& =\left\langle\Pi_{*}\left(f^{n}\right)^{*} \Pi^{*}\left(\omega^{p}\right), \omega_{Y}^{l-p+q} \wedge \omega^{k-l-q}\right\rangle \\
& =\left\langle\left(f^{n}\right)^{*} \Pi^{*}\left(\omega^{p}\right), \Pi^{*}\left(\omega_{Y}^{l-p+q} \wedge \omega^{k-l-q}\right)\right\rangle \\
& =\left\langle\left(f^{n}\right)^{*} \Pi^{*}\left(\omega^{p}\right) \wedge \pi^{*}\left(\omega_{Y}^{l-p+q}\right), \Pi^{*}\left(\omega^{k-l-q}\right)\right\rangle .
\end{aligned}
$$

Observe that

$$
a_{q, p}(n) \geq \alpha_{p-q}\left(\Pi_{*}\left(f^{n}\right)^{*} \Pi^{*}\left(\omega^{p}\right)\right),
$$

where $\alpha_{p-q}(\cdot)$ is defined in (1).

Lemma 4.1. There is a constant $A>0$ independent of $p, n$ such that

$$
A^{-1} \lambda_{p}\left(f^{n} \mid \pi\right) \leq a_{p, p}(n) \leq A \lambda_{p}\left(f^{n} \mid \pi\right)
$$

In particular, $\left[a_{p, p}(n)\right]^{1 / n}$ converges to $d_{p}(f \mid \pi)$.

Proof. Since the pull-back of a smooth form under $\Pi$ is smooth, we have

$$
\begin{aligned}
a_{p, p}(n) & =\left\langle\left(f^{n}\right)^{*} \Pi^{*}\left(\omega^{p}\right) \wedge \pi^{*}\left(\omega_{Y}^{l}\right), \Pi^{*}\left(\omega^{k-l-p}\right)\right\rangle \\
& \leq A\left\langle\left(f^{n}\right)^{*}\left(\omega_{X}^{p}\right) \wedge \pi^{*}\left(\omega_{Y}^{l}\right), \omega_{X}^{k-l-p}\right\rangle=A \lambda_{p}\left(f^{n} \mid \pi\right)
\end{aligned}
$$

for some constant $A>0$. This gives the second inequality in the lemma.

Define $T:=\Pi_{*}\left(\omega_{X}^{p}\right)$. Since $\Pi^{\bullet}(T) \geq \omega_{X}^{p}$, we have

$$
\lambda_{p}(f \mid \pi)=\left\|\left(f^{n}\right)^{*}\left(\omega_{X}^{p}\right) \wedge \pi^{*}\left(\omega_{Y}^{l}\right)\right\| \leq\left\|\left(f^{n}\right)^{\bullet} \Pi^{\bullet}(T) \wedge \pi^{*}\left(\omega_{Y}^{l}\right)\right\| .
$$

We apply Proposition 2.1 to the current $T$ on $Y \times \mathbb{P}^{k-l}$ which is an $L^{1}$ form smooth on a Zariski open set. Let $T_{i}$ be as in that proposition with $\left\{T_{i}\right\} \leq A\left\{\omega^{p}\right\}$ for some constant $A>0$. If $S:=\Pi_{*}\left(\omega_{X}^{k-l-p}\right)$, we have $\Pi^{\bullet}(S) \geq \omega_{X}^{k-l-p}$ and hence

$$
\begin{aligned}
\lambda_{p}(f \mid \pi) & \leq \liminf _{i \rightarrow \infty}\left\|\left(f^{n}\right)^{*} \Pi^{*}\left(T_{i}\right) \wedge \pi^{*}\left(\omega_{Y}^{l}\right)\right\| \\
& \leq A\left\|\left(f^{n}\right)^{*} \Pi^{*}\left(\omega^{p}\right) \wedge \pi^{*}\left(\omega_{Y}^{l}\right)\right\| \\
& =A\left\langle\left(f^{n}\right)^{*} \Pi^{*}\left(\omega^{p}\right) \wedge \pi^{*}\left(\omega_{Y}^{l}\right), \omega_{X}^{k-l-p}\right\rangle \\
& \leq A\left\|\left(f^{n}\right)^{*} \Pi^{*}\left(\omega^{p}\right) \wedge \pi^{*}\left(\omega_{Y}^{l}\right) \wedge \Pi^{\bullet}(S)\right\| .
\end{aligned}
$$


Vol. 86 (2011) Comparison of dynamical degrees for semi-conjugate meromorphic maps 833

Now, we apply again Proposition 2.1, in particular its last assertion, to the current $S$ which is an $L^{1}$ form smooth on a Zariski open set. If $S_{i}$ are smooth forms satisfying that proposition, the latter expression is bounded from above by

$$
\begin{aligned}
& \liminf _{i \rightarrow \infty}\left\langle\left(f^{n}\right)^{*} \Pi^{*}\left(\omega^{p}\right) \wedge \pi^{*}\left(\omega_{Y}^{l}\right), \Pi^{*}\left(S_{i}\right)\right\rangle \\
& \quad \lesssim\left\langle\left(f^{n}\right)^{*} \Pi^{*}\left(\omega^{p}\right) \wedge \pi^{*}\left(\omega_{Y}^{l}\right), \Pi^{*}\left(\omega^{k-l-p}\right)\right\rangle .
\end{aligned}
$$

The last integral is equal to $a_{p, p}(n)$. The first inequality in the lemma follows.

Define for $0 \leq p \leq k$

$$
b_{p}(n):=\sum_{\max \{0, p-l\} \leq q \leq \min \{p, k-l\}} a_{q, p}(n) .
$$

We have the following lemma.

Lemma 4.2. The sequence $b_{p}(n)^{1 / n}$ converges to $d_{p}(f)$.

Proof. Since $\Pi^{*}\left(\omega^{p}\right), \pi^{*}\left(\omega_{Y}^{l-p+q}\right)$ and $\Pi^{*}\left(\omega^{k-l-q}\right)$ are smooth on $X$, we have

$$
\begin{aligned}
a_{q, p}(n) & =\left\langle\left(f^{n}\right)^{*} \Pi^{*}\left(\omega^{p}\right) \wedge \pi^{*}\left(\omega_{Y}^{l-p+q}\right), \Pi^{*}\left(\omega^{k-l-q}\right)\right\rangle \\
& \leq A\left\|\left(f^{n}\right)^{*}\left(\omega_{X}^{p}\right)\right\|=A \lambda_{p}\left(f^{n}\right)
\end{aligned}
$$

for some constant $A>0$. We deduce that $\lim \sup b_{p}(n)^{1 / n} \leq d_{p}(f)$.

It remains to check that $\lim \inf b_{p}(n)^{1 / n} \geq d_{p}(f)$. For this purpose, we only need to show that $\lambda_{p}\left(f^{n}\right) \leq A b_{p}(n)$ for some constant $A>0$. Define $T:=$ $\Pi_{*}\left(f^{n}\right)^{*} \Pi^{*}\left(\omega^{p}\right)$. We prove that $\lambda_{p}\left(f^{n}\right) \lesssim\|T\| \lesssim b_{p}(n)$ which will imply the result.

Define $S:=\Pi_{*}\left(\omega_{X}^{p}\right)$. We have $\Pi^{\bullet}(S) \geq \omega_{X}^{p}$. Therefore,

$$
\lambda_{p}\left(f^{n}\right)=\left\langle\left(f^{n}\right)^{*}\left(\omega_{X}^{p}\right), \omega_{X}^{k-p}\right\rangle \leq\left\langle\left(f^{n}\right)^{\bullet} \Pi^{\bullet}(S), \omega_{X}^{k-p}\right\rangle .
$$

Using a semi-regularization of $S$, we deduce that

$$
\lambda_{p}\left(f^{n}\right) \lesssim\left\langle\left(f^{n}\right)^{*} \Pi^{*}\left(\omega^{p}\right), \omega_{X}^{k-p}\right\rangle .
$$

Define $R:=\Pi_{*}\left(\omega_{X}^{k-p}\right)$. We also have $\Pi^{\bullet}(R) \geq \omega_{X}^{k-p}$. We obtain as above using a semi-regularization of $R$ that

$$
\begin{aligned}
\lambda_{p}\left(f^{n}\right) & \lesssim\left\|\left(f^{n}\right)^{*} \Pi^{*}\left(\omega^{p}\right) \stackrel{\circ}{\wedge} \Pi^{\bullet}(R)\right\| \lesssim\left\|\left(f^{n}\right)^{*} \Pi^{*}\left(\omega^{p}\right) \wedge \Pi^{*}\left(\omega^{k-p}\right)\right\| \\
& =\left\langle\Pi_{*}\left(f^{n}\right)^{*} \Pi^{*}\left(\omega^{p}\right), \omega^{k-p}\right\rangle=\|T\| .
\end{aligned}
$$


Now, since $\omega_{Y}^{l+1}=0$ and $\omega_{\mathrm{FS}}^{k-l+1}=0$, we have

$$
\begin{aligned}
\|T\| & =\left\langle T,\left(\omega_{Y}+\omega_{\mathrm{FS}}\right)^{k-p}\right\rangle \lesssim \sum_{\max \{0, p-l\} \leq q \leq \min \{p, k-l\}}\left\langle T, \omega_{Y}^{l-p+q} \wedge \omega_{\mathrm{FS}}^{k-l-q}\right\rangle \\
& \leq \sum_{\max \{0, p-l\} \leq q \leq \min \{p, k-l\}} a_{q, p}(n)=b_{p}(n) .
\end{aligned}
$$

This completes the proof of the lemma.

For every $n \geq 0$ and $0 \leq p \leq l$ define

$$
c_{p}(n):=\lambda_{p}\left(g^{n}\right)=\left\|\left(g^{n}\right)^{*}\left(\omega_{Y}^{p}\right)\right\|=\left\langle\left(g^{n}\right)^{*}\left(\omega_{Y}^{p}\right), \omega_{Y}^{l-p}\right\rangle .
$$

We have the following lemma.

Lemma 4.3. There is a constant $A>0$ such that

$$
\left\langle\Pi_{*}\left(f^{n}\right)^{*} \Pi^{*}\left(\omega_{Y}^{p-q} \wedge \omega^{q}\right), \omega_{Y}^{l-p+p_{0}} \wedge \omega^{k-l-p_{0}}\right\rangle \leq A a_{p_{0}, q}(n) c_{p-q}(n)
$$

for $0 \leq p_{0} \leq k-l, p_{0} \leq p \leq l+p_{0}, p_{0} \leq q \leq p$ and $n \geq 0$. Moreover, the above integral vanishes when $q<p_{0}$.

Proof. Observe that by definition of $\Pi_{*}$

$$
\begin{aligned}
\Pi_{*}\left(f^{n}\right)^{*} \Pi^{*}\left(\omega_{Y}^{p-q} \wedge \omega^{q}\right) & =\Pi_{*}\left[\left(f^{n}\right)^{*} \Pi^{*}\left(\omega_{Y}^{p-q}\right) \stackrel{\circ}{\wedge}\left(f^{n}\right)^{*} \Pi^{*}\left(\omega^{q}\right)\right] \\
& \leq \Pi_{*}\left(f^{n}\right)^{*} \Pi^{*}\left(\omega_{Y}^{p-q}\right) \stackrel{\circ}{\wedge} \Pi_{*}\left(f^{n}\right)^{*} \Pi^{*}\left(\omega^{q}\right) .
\end{aligned}
$$

Hence, the left hand side of the inequality in the lemma is smaller than or equal to

$$
\left\langle\Pi_{*}\left(f^{n}\right)^{*} \Pi^{*}\left(\omega_{Y}^{p-q}\right) \stackrel{\circ}{\wedge} \Pi_{*}\left(f^{n}\right)^{*} \Pi^{*}\left(\omega^{q}\right), \omega_{Y}^{l-p+p_{0}} \wedge \omega^{k-l-p_{0}}\right\rangle .
$$

Define $T:=\Pi_{*}\left(f^{n}\right)^{*} \Pi^{*}\left(\omega_{Y}^{p-q}\right) \wedge \omega_{Y}^{l-p+p_{0}}$ and $S:=\Pi_{*}\left(f^{n}\right)^{*} \Pi^{*}\left(\omega^{q}\right) \wedge \omega^{k-l-p_{0}}$. Note that $T$ and $S$ are of bidegree $\left(l-q+p_{0}, l-q+p_{0}\right)$ and $\left(k-l+q-p_{0}, k-\right.$ $\left.l+q-p_{0}\right)$ respectively. The quantity considered above is equal to the mass of the measure $T \stackrel{\circ}{\wedge} S$.

We first show that $\alpha_{j}(T)=0$ when $j<l-q+p_{0}$ and $\alpha_{l-q+p_{0}}(T) \leq A c_{p-q}(n)$ for some constant $A>0$. Since $\pi \circ f^{n}=g^{n} \circ \pi$, we have

$$
T=\Pi_{*}\left(f^{n}\right)^{*} \pi^{*}\left(\omega_{Y}^{p-q}\right) \wedge \omega_{Y}^{l-p+p_{0}}=\Pi_{*} \pi^{\bullet}\left(g^{n}\right)^{*}\left(\omega_{Y}^{p-q}\right) \wedge \omega_{Y}^{l-p+p_{0}} .
$$

Hence,

$$
\begin{aligned}
\alpha_{j}(T) & =\left\langle\Pi_{*} \pi^{\bullet}\left(g^{n}\right)^{*}\left(\omega_{Y}^{p-q}\right) \wedge \omega_{Y}^{l-p+p_{0}}, \omega_{Y}^{l-j} \wedge \omega_{\mathrm{FS}}^{k-2 l+q-p_{0}+j}\right\rangle \\
& =\left\langle\pi^{\bullet}\left(g^{n}\right)^{*}\left(\omega_{Y}^{p-q}\right) \wedge \pi^{*}\left(\omega_{Y}^{l-p+p_{0}}\right), \pi^{*}\left(\omega_{Y}^{l-j}\right) \wedge \Pi^{*}\left(\omega_{\mathrm{FS}}^{k-2 l+q-p_{0}+j}\right)\right\rangle \\
& =\left\langle\pi^{\bullet}\left[\left(g^{n}\right)^{*}\left(\omega_{Y}^{p-q}\right) \wedge \omega_{Y}^{2 l-p+p_{0}-j}\right], \Pi^{*}\left(\omega_{\mathrm{FS}}^{k-2 l+q-p_{0}+j}\right)\right\rangle .
\end{aligned}
$$


Vol. 86 (2011) Comparison of dynamical degrees for semi-conjugate meromorphic maps 835

When $j<l-q+p_{0}$, the form in the brackets has bidegree $\geq(l+1, l+1)$ and should vanish because $\operatorname{dim} Y=l$. Therefore, $\alpha_{j}(T)=0$ in that case. When $j=l-q+p_{0}$, this form defines a positive measure of mass $\lambda_{p-q}\left(g^{n}\right)$. Its cohomology class is equal to $\lambda_{p-q}\left(g^{n}\right)\left\{\omega_{Y}^{l}\right\}$. Therefore, using a semi-regularization as above, we obtain

$$
\alpha_{l-q+p_{0}}(T) \lesssim \lambda_{p-q}\left(g^{n}\right)\left\langle\pi^{*}\left(\omega_{Y}^{l}\right), \Pi^{*}\left(\omega_{\mathrm{FS}}^{k-l}\right)\right\rangle \leq A c_{p-q}(n)
$$

for some constant $A>0$.

We deduce from Proposition 2.3 that $\{T\} \lesssim c_{p-q}(n)\left\{\omega_{Y}^{l-q+p_{0}}\right\}$. Using the semiregularization in Proposition 2.4 for $T$, we obtain

$$
\{T \stackrel{\circ}{\wedge} S\} \lesssim c_{p-q}(n)\left\|\omega_{Y}^{l-q+p_{0}} \wedge S\right\|=c_{p-q}(n) a_{p_{0}, q}(n)
$$

This completes the proof of the first assertion in the lemma. For the second one, it is enough to observe that when $q<p_{0}$, we have $\alpha_{j}(T)=0$ for every $j$ and hence $T=0$.

The following lemma is crucial in our proof.

Lemma 4.4. There exists a constant $A>0$ such that for all $0 \leq p_{0} \leq k-l$, $p_{0} \leq p \leq l+p_{0}$ and all $n, r \geq 1$

$$
a_{p_{0}, p}(n r) \leq A^{r} \sum \prod_{s=1}^{r} a_{p_{s-1}, p_{s}}(n) c_{p-p_{s}}(n),
$$

where the sum is taken over $\left(p_{1}, \ldots, p_{r}\right)$ with $p_{0} \leq p_{1} \leq p_{2} \leq \cdots \leq p_{r} \leq p$ and $p_{r-1} \leq k-l$.

Proof. We proceed by induction on $r$. Clearly, the lemma is true for $r=1$. Suppose the lemma true for $r$, we need to prove it for $r+1$. In what follows, the constants $A_{i}$ depend only on the geometry of $X$ and $Y$.

Define $T^{(r)}:=\Pi_{*}\left(f^{n r}\right)^{*} \Pi^{*}\left(\omega^{p}\right)$. This is a positive closed $L^{1}$ form, smooth on a dense Zariski open set. Observe that $\Pi^{\bullet} \Pi_{*} \geq$ id on positive closed currents having no mass on proper analytic subsets of $X$. Therefore,

$$
T^{(r+1)} \leq \Pi_{*}\left(f^{n}\right)^{\bullet} \Pi^{\bullet} \Pi_{*}\left(f^{n r}\right)^{\bullet} \Pi^{*}\left(\omega^{p}\right)=\Pi_{*}\left(f^{n}\right)^{\bullet} \Pi^{\bullet}\left(T^{(r)}\right) .
$$

On the other hand, by Proposition 2.4, we can find a sequence of smooth positive closed $(p, p)$-forms $T_{i}^{(r)}$ converging weakly to a positive closed current $\tilde{T}^{(r)} \geq T^{(r)}$ such that

$$
\alpha_{p-q}\left(T_{i}^{(r)}\right) \leq A_{1} \alpha_{p-q}\left(T^{(r)}\right) \leq A_{1} a_{q, p}(n r)
$$


for $\max \{0, p-l\} \leq q \leq \min \{p, k-l\}$ and $A_{1}>0$ a constant. By Proposition 2.3, there is a constant $A_{2}>0$ such that

$$
\left\{T_{i}^{(r)}\right\} \leq A_{2} \sum_{\max \{0, p-l\} \leq q \leq \min \{p, k-l\}} a_{q, p}(n r)\left\{\omega_{Y}^{p-q}\right\} \cup\left\{\omega_{\mathrm{FS}}^{q}\right\} .
$$

We deduce from the above discussion and Lemma 4.3 that

$$
\begin{aligned}
a_{p_{0}, p} & (n(r+1))=\left\langle T^{(r+1)}, \omega_{Y}^{l-p+p_{0}} \wedge \omega^{k-l-p_{0}}\right\rangle \\
\leq & \liminf _{i \rightarrow \infty}\left\langle\Pi_{*}\left(f^{n}\right)^{*} \Pi^{*}\left(T_{i}^{(r)}\right), \omega_{Y}^{l-p+p_{0}} \wedge \omega^{k-l-p_{0}}\right\rangle \\
\leq & A_{2} \sum_{\substack{\max \{0, p-l\} \leq q \\
\leq \min \{p, k-l\}}} a_{q, p}(n r)\left\langle\Pi_{*}\left(f^{n}\right)^{*} \Pi^{*}\left(\omega_{Y}^{p-q} \wedge \omega_{\mathrm{FS}}^{q}\right), \omega_{Y}^{l-p+p_{0}} \wedge \omega^{k-l-p_{0}}\right\rangle \\
\leq & A_{3} \sum_{p_{0} \leq q \leq \min \{p, k-l\}} a_{q, p}(n r) a_{p_{0}, q}(n) c_{p-q}(n)
\end{aligned}
$$

for some constant $A_{3}>0$. Consequently, the induction hypothesis implies the result.

Theorem 1.1 is a consequence of the next two propositions.

Proposition 4.5. We have

$$
d_{p}(f) \geq \max _{\max \{0, p-k+l\} \leq j \leq \min \{p, l\}} d_{j}(g) d_{p-j}(f \mid \pi)
$$

for $0 \leq p \leq k$

Proof. Since $\Pi^{*}\left(\omega_{Y}^{j} \wedge \omega^{p-j}\right)$ is a smooth form, we have for some constant $A>0$

$$
\left\|\left(f^{n}\right)^{*} \Pi^{*}\left(\omega_{Y}^{j} \wedge \omega^{p-j}\right)\right\| \leq A \lambda_{p}\left(f^{n}\right) .
$$

So, by definition of dynamical degrees and Lemma 4.1, it is enough to bound $\left\|\left(f^{n}\right)^{*} \Pi^{*}\left(\omega_{Y}^{j} \wedge \omega^{p-j}\right)\right\|$ from below by a constant times $\lambda_{j}\left(g^{n}\right) a_{p-j, p-j}(n)$.

Fix a constant $A>0$ large enough. Using the identity $\pi \circ f^{n}=g^{n} \circ \pi$ and that $\Pi^{*}\left(\omega_{Y}^{l-j} \wedge \omega^{k-l-p+j}\right)$ is smooth, we obtain

$$
\begin{aligned}
A\left\|\left(f^{n}\right)^{*} \Pi^{*}\left(\omega_{Y}^{j} \wedge \omega^{p-j}\right)\right\| \\
\quad \geq\left\langle\left(f^{n}\right)^{*} \Pi^{*}\left(\omega_{Y}^{j} \wedge \omega^{p-j}\right), \Pi^{*}\left(\omega_{Y}^{l-j} \wedge \omega^{k-l-p+j}\right)\right\rangle \\
\quad=\left\langle\left(f^{n}\right)^{*} \pi^{*}\left(\omega_{Y}^{j}\right) \wedge\left(f^{n}\right)^{*} \Pi^{*}\left(\omega^{p-j}\right), \pi^{*}\left(\omega_{Y}^{l-j}\right) \wedge \Pi^{*}\left(\omega^{k-l-p+j}\right)\right\rangle \\
\quad=\left\|\left(f^{n}\right)^{*} \pi^{*}\left(\omega_{Y}^{j}\right) \wedge \pi^{*}\left(\omega_{Y}^{l-j}\right) \wedge\left(f^{n}\right)^{*} \Pi^{*}\left(\omega^{p-j}\right) \wedge \Pi^{*}\left(\omega^{k-l-p+j}\right)\right\| \\
\quad=\left\|\pi^{*}\left[\left(g^{n}\right)^{*}\left(\omega_{Y}^{j}\right) \wedge \omega_{Y}^{l-j}\right] \wedge\left(f^{n}\right)^{*} \Pi^{*}\left(\omega^{p-j}\right) \wedge \Pi^{*}\left(\omega^{k-l-p+j}\right)\right\| .
\end{aligned}
$$


Vol. 86 (2011) Comparison of dynamical degrees for semi-conjugate meromorphic maps 837

Observe that $\left(g^{n}\right)^{*}\left(\omega_{Y}^{j}\right) \wedge \omega_{Y}^{l-j}$ is a positive measure of mass $\lambda_{j}\left(g^{n}\right)$. As in Lemma 3.2, we show that the last expression in the previous identities is equal to $\lambda_{j}\left(g^{n}\right)$ times the mass of the restriction of $\left(f^{n}\right)^{*} \Pi^{*}\left(\omega^{p-j}\right) \wedge \Pi^{*}\left(\omega^{k-l-p+j}\right)$ to a generic fiber $L_{y}$ of $\pi$. Therefore, it is also equal to

$$
\lambda_{j}\left(g^{n}\right)\left\langle\pi^{*}\left(\omega_{Y}^{l}\right),\left(f^{n}\right)^{*} \Pi^{*}\left(\omega^{p-j}\right) \wedge \Pi^{*}\left(\omega^{k-l-p+j}\right)\right\rangle=\lambda_{j}\left(g^{n}\right) a_{p-j, p-j}(n) .
$$

This completes the proof.

Proposition 4.6. We have

$$
d_{p}(f) \leq \max _{\max \{0, p-k+l\} \leq j \leq \min \{p, l\}} d_{j}(g) d_{p-j}(f \mid \pi)
$$

for $0 \leq p \leq k$

Proof. For every $0 \leq p \leq k$ and $n \geq 0$ let

$$
\mu_{p}(n):=\max _{\max \{0, p-k+l\} \leq j \leq \min \{p, l\}} c_{j}(n) a_{p-j, p-j}(n) .
$$

Observe that for $r>p$, in Lemma 4.4, there are at most $p$ indices $s$ such that $p_{s-1}<p_{s}$. Moreover, the sum in that lemma contains at most $(k+1)^{r}$ terms and the sum in the definition of $b_{p}(n)$ contains at most $p+1$ terms. We infer the following estimate

$$
b_{p}(r n) \leq\left[(p+1)(k+1)^{r} A^{r} b_{0}(n) \cdots b_{p}(n) \prod_{j=0}^{l} c_{j}(n)\right] \mu_{p}(n)^{r} .
$$

We deduce that

$$
\begin{aligned}
{\left[b_{p}(r n)\right]^{1 / r n} \leq(p+1)^{1 / n r}(k+1)^{1 / n} A^{1 / n}\left[b_{0}(n)^{1 / n} \cdots b_{p}(n)^{1 / n}\right]^{1 / r} } \\
\cdot\left[\prod_{j=0}^{l} c_{j}(n)^{1 / n}\right]^{1 / r} \mu_{p}(n)^{1 / n} .
\end{aligned}
$$

Letting $n$ tend to infinity, we obtain using Lemma 4.2 that

$$
d_{p}(f) \leq\left[d_{0}(f) \ldots d_{p}(f)\right]^{1 / r}\left[\prod_{j=0}^{l} d_{j}(g)\right]^{1 / r} \liminf _{n \rightarrow \infty} \mu_{p}(n)^{1 / n}
$$

Now, letting $r \rightarrow \infty$, the first two factors in the right hand side tend to 1 . Therefore, using Lemma 4.1, we obtain

$$
d_{p}(f) \leq \liminf _{n \rightarrow \infty} \mu_{p}(n)^{1 / n}=\max _{\max \{0, p-k+l\} \leq j \leq \min \{p, l\}} d_{j}(g) d_{p-j}(f \mid \pi) .
$$

This completes the proof. 
Proof of Corollary 1.2. When $X$ and $Y$ are projective, the corollary is a direct consequence of Theorem 1.1. We only used the projectivity in Proposition 2.4 applied to $m:=k-l$ and for the existence of $v: X \rightarrow \mathbb{P}^{k-l}$. This is superfluous when $X$ and $Y$ have the same dimension, i.e. $k=l$.

Proof of Corollary 1.3. Let $j, p$ be such that $d_{j}(g)=\max _{q} d_{q}(g)$ and $d_{p-j}(f \mid \pi)=$ $\max _{q} d_{q}(f \mid \pi)$. We have $0 \leq j \leq l$ and $0 \leq p-j \leq k-l$. By Theorem 1.1, $d_{p}(f)$ is the maximal dynamical degree of $f$ and $d_{p}(f)=d_{j}(g) d_{p-j}(f \mid \pi)$. We have $d_{p-1}(f)<d_{p}(f)<d_{p+1}(f)$. Theorem 1.1 implies that

$d_{j-1}(g)<d_{j}(g)<d_{j+1}(g)$ and $\quad d_{p-j-1}(f \mid \pi)<d_{p-j}(f \mid \pi)<d_{p-j+1}(f \mid \pi)$.

The log-concavity of $d_{q}(g)$ and $d_{q}(f \mid \pi)$ implies the result. Note that when $j=0, l$ or $p-j=0, k-l$, in the above inequalities, one has to remove the expressions which are not meaningful.

In the rest of the paper, we prove Corollary 1.4. Let $K_{X}$ denote the canonical lines bundle of $X$. Let $H^{0}\left(X, K_{X}^{n}\right)$ denote the space of holomorphic sections of $K_{X}^{n}$ and $H^{0}\left(X, K_{X}^{n}\right)^{*}$ its dual space. Assume that $H^{0}\left(X, K_{X}^{n}\right)$ has positive dimension. If $x$ is a generic point in $X$, the family $H_{x}$ of sections which vanish at $x$ is a hyperplane of $H^{0}\left(X, K_{X}^{n}\right)$ passing through 0 . So, the correspondence $x \mapsto H_{x}$ defines a meromorphic map

$$
\pi_{n}: X \rightarrow \mathbb{P} H^{0}\left(X, K_{X}^{n}\right)^{*}
$$

from $X$ to the projectivization of $H^{0}\left(X, K_{X}^{n}\right)^{*}$ which is called an Iitaka fibration of $X$. Let $Y_{n}$ denote the image of $X$ by $\pi_{n}$. The Kodaira dimension of $X$ is $\kappa_{X}:=$ $\max _{n \geq 1} \operatorname{dim} Y_{n}$. When $H^{0}\left(X, K_{X}^{n}\right)=0$ for every $n \geq 1$, the Kodaira dimension of $X$ is $-\infty$. We have the following result.

Theorem 4.7 ([14], [18]). Let $f: X \rightarrow X$ be a dominant meromorphic map. Assume that $\kappa_{X} \geq 1$. Then $f$ preserves the Iitaka fibration $\pi_{n}: X \rightarrow Y_{n}$. Moreover, the map $g: Y_{n} \rightarrow Y_{n}$ induced by $f$ is periodic, i.e. $g^{N}=$ id for some integer $N \geq 1$.

Proof of Corollary 1.4. Assume in order to get a contradiction that $\kappa_{X} \geq 1$. Let $n \geq 1$ be such that $l:=\operatorname{dim} Y_{n} \geq 1$. Replacing $f$ with an iterate, we can assume that $g=$ id. A priori, $Y_{n}$ may be singular, but we can use a blow-up and assume that $Y_{n}$ is smooth. We have $d_{j}(g)=1$ for $0 \leq j \leq l$. This contradicts Corollary 1.3. Note that in order to prove that $d_{j}(g)=1$, instead of Theorem 4.7, it is enough to use the weaker result that $g$ is induced by a linear endomorphism of $\mathbb{P} H^{0}\left(X, K_{X}^{n}\right)^{*}$. 
Vol. 86 (2011) Comparison of dynamical degrees for semi-conjugate meromorphic maps 839

\section{References}

[1] E. Amerik and F. Campana, Fibrations méromorphes sur certaines variétés à fibré canonique trivial. Pure Appl. Math. Q. 4 (2008), no. 2, part 1, 509-545. Zbl 1143.14035 MR 1143.14035

[2] A. Blanchard, Sur les variétés analytiques complexes. Ann. Sci. École Norm. Sup. (3) 73 (1956), 157-202. Zbl 0073.37503 MR 0087184

[3] S. Cantat, Dynamique des automorphismes des surfaces projectives complexes. C. R. Acad. Sci. Paris Sér. I Math. 328 (10) (1999), 901-906. Zbl 0943.37021 MR 1689873

[4] J.-P. Demailly, Complex analytic and differential geometry. Available at http://www-fourier.ujf-grenoble.fr/ demailly/manuscripts/agbook.pdf.

[5] T.-C. Dinh, Suites d'applications méromorphes multivaluées et courants laminaires. $J$. Geom. Anal. 15 (2005), no. 2, 207-227. Zbl 1085.37039 MR 2152480

[6] T.-C. Dinh and N. Sibony, Une borne supérieure pour l'entropie topologique d'une application rationnelle. Ann. of Math. (2) 161 (2005), 1637-1644. Zbl 1084.54013 MR 2180409

[7] T.-C. Dinh and N. Sibony, Regularization of currents and entropy. Ann. Sci. École Norm. Sup. (4) 37 (2004), no. 6, 959-971. Zbl 1074.53058 MR 2119243

[8] T.-C. Dinh and N. Sibony, Dynamics in several complex variables: endomorphisms of projective spaces and polynomial-like mappings. In Holomorphic dynamical systems, Lecture Notes in Math. 1998, Springer-Verlag, Berlin 2010, 165-294. Zbl 05879453 MR 2648690

[9] M. Gromov, Convex sets and Kähler manifolds. In Advances in differential geometry and topology, World Scientific Publishing Co., Inc., Teaneck, NJ, 1998, 1-38. Zbl 0770.53042 MR 1095529

[10] M. Gromov, On the entropy of holomorphic maps. Enseign. Math. 49 (2003), 217-235. Zbl 1080.37051 MR 2026895

[11] V. Guedj, Propriétés ergodiques des applications rationnelles. Panoramas \& Synthèses, to appear; arXiv:math/0611302 [math.CV].

[12] B. Hasselblatt and J. Propp, Degree-growth of monomial maps. Ergodic Theory Dynam. Systems 27 (2007), no. 5, 1375-1397. Zbl 1143.37032 MR 2358970

[13] A. G. Khovanskii, The geometry of convex polyhedra and algebraic geometry. Uspehi Mat. Nauk. 34 (1979), no. 4, 160-161.

[14] N. Nakayama and D.-Q. Zhang, Building blocks of etale endomorphisms of complex projective manifolds. Proc. London Math. Soc. 99 (2009), 725-756. Zbl 1185.14012 MR 2551469

[15] N. Sibony, Dynamique des applications rationnelles de $\mathbb{P}^{k}$. In Dynamique et géométrie complexes (Lyon, 1997), Panoramas et Synthèses 8, Soc. Math. France, Paris 1999, 97-185. Zbl 1020.37026 MR 1760844

[16] H. Skoda, Prolongement des courants positifs, fermés de masse finie. Invent. Math. 66 (1982), 361-376. Zbl 0488.58002 MR 0662596

[17] B. Teissier, Du théorème de l'index de Hodge aux inégalités isopérimétriques. C. R. Acad. Sci. Paris Sér. A-B 288 (1979), no. 4, 287-289. Zbl 0406.14011 MR 0524795 
[18] K. Ueno, Classification theory of algebraic varieties and compact complex spaces. Notes written in collaboration with P. Cherenack, Lecture Notes in Math. 439, Springer-Verlag, Berlin 1975. Zbl 0299.14007 MR 0506253

[19] C. Voisin, Hodge theory and complex algebraic geometry. I. Cambridge Stud. Adv. Math. 76, Cambridge University Press, Cambridge 2002. Zbl 1005.14002 MR 2451566

[20] Y. Yomdin, Volume growth and entropy. Israel J. Math., 57 (1987) 285-300. Zbl 0641.54036 MR 0889979

[21] D.-Q. Zhang, Cohomologically hyperbolic endomorphisms of complex manifolds. Internat. J. Math. 20 (2009), 803-816. Zbl 1172.14009 MR 2548399

[22] D.-Q. Zhang, Polarized endomorphisms of uniruled varieties (with an Appendix by Y. Fujimoto and N. Nakayama). Compos. Math. 146 (2010), no. 1, 145-168. Zbl 05706184 MR 2581245

Received March 29, 2009

Tien-Cuong Dinh, UPMC, Université Paris 06, UMR 7586, Institut de Mathématiques de Jussieu, 4 place Jussieu, 75005 Paris, France

E-mail: dinh@math.jussieu.fr

http://www.math.jussieu.fr/ dinh

Viêt-Anh Nguyên, Équipe Analyse Harmonique, Mathématiques, Bâtiment 425, Université Paris-Sud 11, 91405 Orsay Cedex, France

E-mail: vietanh.nguyen@ math.u-psud.fr

http://www.math.u-psud.fr/ vietanh 